\title{
Clinical Application of 3D-FIESTA Image in Patients with Unilateral Inner Ear Symptom
}

\author{
Jae Ho Oh, Jae Ho Chung, Hyun Jung Min, \\ Seok Hyun Cho, Chul Won Park and Seung Hwan Lee \\ Department of Otolaryngology-Head and Neck Surgery, College of Medicine, Hanyang University, Seoul, Korea
}

Received July 10, 2013

Revised September 20, 2013

Accepted October 13, 2013

Address for correspondence

Seung Hwan Lee, MD

Department of Otolaryngology-

Head and Neck Surgery,

College of Medicine,

Hanyang University,

222 Wangsimni-ro, Seongdong-gu,

Seoul 133-792, Korea

Tel $+82-31-560-2363$

Fax +82-31-566-4884

E-mail shleemd@hanyang.ac.kr
Background and Objectives: Unilateral auditory dysfunction such as tinnitus and hearing loss could be a warning sign of a retrocochlear lesion. Auditory brainstem response (ABR) and internal auditory canal magnetic resonance image (MRI) are suggested as novel diagnostic tools for retrocochlear lesions. However, the high cost of MRI and the low sensitivity of the ABR test could be an obstacle when assessing patients with unilateral ear symptoms. The purpose of this study was to introduce the clinical usefulness of three-dimensional fast imaging employing steady-state acquisition (3D-FIESTA) MRI in patients with unilateral ear symptoms. Subjects and Methods: Two hundred and fifty-three patients with unilateral tinnitus or unilateral hearing loss who underwent 3D-FIESTA temporal bone MRI as a screening test were enrolled. We reviewed the abnormal findings in the 3D-FIESTA images and ear symptoms using the medical records. Results: In patients with unilateral ear symptoms, $51.0 \%$ of the patients had tinnitus and $32.8 \%$ patients were assessed to have sudden sensory neural hearing loss. With 3D-FIESTA imaging, twelve patients were diagnosed with acoustic neuroma, four with enlarged vestibular aqueduct syndrome, and two with posterior inferior cerebellar artery aneurysm. Inner ear anomalies and vestibulocochlear nerve aplasia could be diagnosed with 3D-FIESTA imaging. Conclusions: 3D-FIESTA imaging is a highly sensitive method for the diagnosis of cochlear or retrocochlear lesions. 3D-FIESTA imaging is a useful screening tool for patients with unilateral ear symptoms.

Korean J Audiol 2013;17:111-117

\section{Introduction}

It is very important to identify the underlying cause of ear symptoms such as tinnitus and hearing loss. If these symptoms present unilaterally, a retocochlear lesion, vascular lesion or inner ear anomaly should be considered in the diagnostic process. Many diagnostic tools have been suggested for use in the evaluation of retrocochlear lesions. However, there is no single novel method to easily evaluate all lesions causing unilateral ear symptoms.

Since Selters first introduced auditory brainstem evoked response (ABR) as a diagnostic method for retrocochlear le-

This is an Open Access article distributed under the terms of the Creative Commons Attribution Non-Commercial License (http://creativecommons. org/licenses/by-nc/3.0/) which permits unrestricted non-commercial use, distribution, and reproduction in any medium, provided the original work is properly cited. sions, the ABR test has been commonly used as a screening test. However, the low sensitivity of the ABR test has lowered its diagnostic value as a screening test, especially in small intracanalicular tumors. ${ }^{1,2)}$

After the introduction of magnetic resonance image (MRI) technology, MRI testing was applied in the assessment of retrocochlear lesions with high sensitivity and high specificity. ${ }^{3)}$ Nevertheless, the high cost of conventional MRI testing, the use of contrast media and the long image acquisition time might be limitations for using it as a screening tool.

Recently, three-dimensional fast imaging employing steadystate acquisition (3D-FIESTA) images have been introduced. 3D-FIESTA imaging can give much higher spatial resolution with outstanding image contrast between the cranial nerves and cerebrospinal fluid. And it has a shorter image acquisition time than conventional MRI scan and does not need contrast 
media., $^{4,5}$

In this study, we aimed to evaluate the usability of 3D-FIESTA imaging as a screening tool for inner ear lesions with applying 3D-FIESTA image in the diagnostic pathway of the patients with unilateral ear symptoms.

\section{Subjects and Methods}

From January 2011 to December 2011, 253 patients who presented with unilateral ear symptoms and underwent 3D-FIESTA imaging as a screening were enrolled in this study. Patients suspected to have Meniere's disease and complained of bilateral ear symptoms were excluded. A thorough medical review of the clinical presentation and audiometric results was performed retrospectively.

All patients underwent 3D-FIESTA imaging as a screening tool with a Philips Achieva (Best, the Netherlands) 3.0 tesla MRI scanner. If an inner ear lesion or a brain lesion was suspected on the 3D-FIESTA image, an additional diagnostic

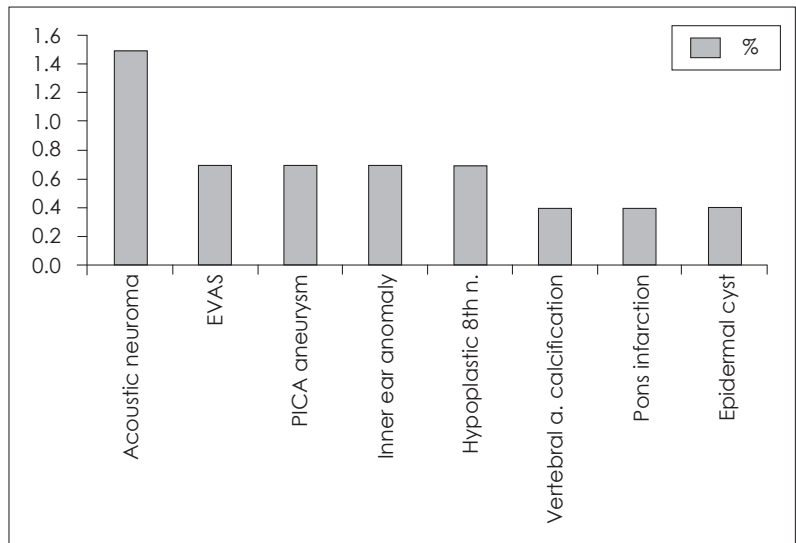

Fig. 1. Prevalence of each disease among patients with unilateral ear symptoms. EVAS: enlarged vestibular aqueduct syndrome, PICA: posterior inferior cerebellar artery.
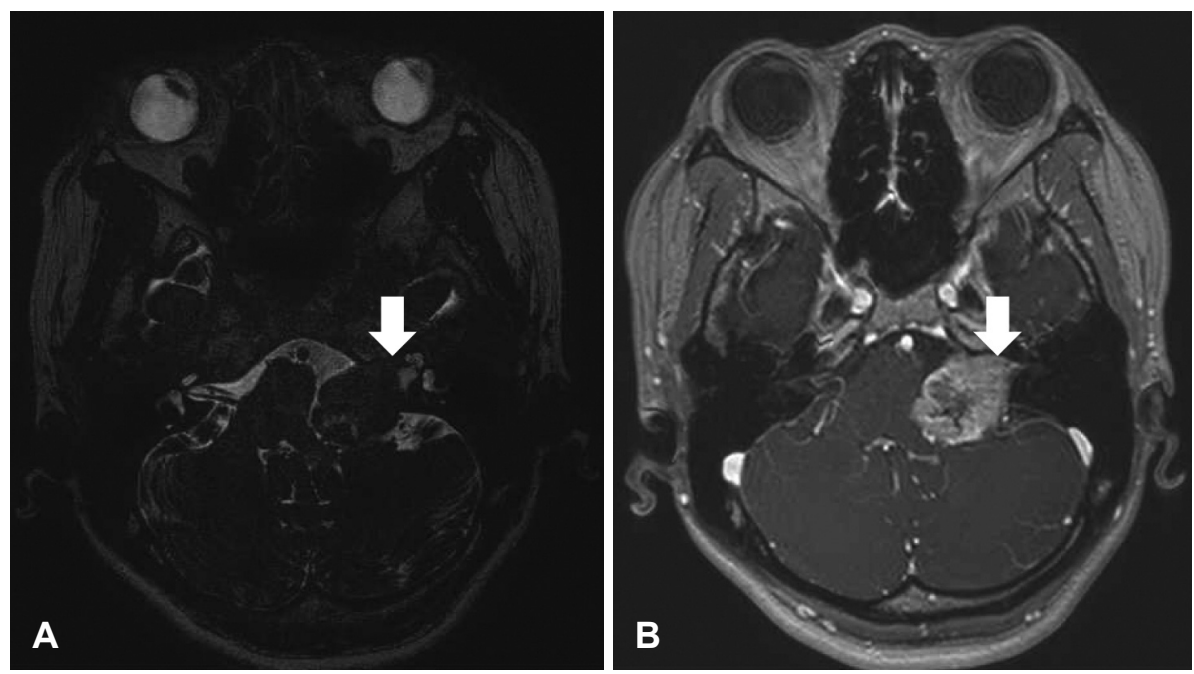

Fig. 2. Temporal bone images of a 21 year old woman with left sudden hearing loss. Acoustic neuroma extending to the cerebellopontine angle (arrows) was identified by screening using $3 \mathrm{D}$ fiesta imaging $(\mathrm{A})$ and was confirmed by Gd enhanced T1weighted imaging (B). 3D: three-dimensional. 
We also tried to figure out the association between clinical symptoms and the final diagnosis. Six patients who initially presented with tinnitus were diagnosed with acoustic neuroma and two patients were diagnosed with posterior inferior cerebellar artery aneurysm. Abnormal findings in 3D-FIESTA images in patients with hearing loss were acoustic neuroma, enlarged vestibular aqueduct syndrome, inner ear anomaly and 8th nerve hypoplasia (Table 1).

The mean size of acoustic neuromas which were finally diagnosed was $17 \times 14 \mathrm{~mm}(4 \times 3-62 \times 64 \mathrm{~mm})$, the mean size of tumors in the tinnitus group, unilateral hearing loss group and sudden idiopathic sensorineural hearing loss group

Fig. 3. Temporal bone images of a 69 year old woman with tinnitus on the right side. An intracanalicular acoustic neuroma (arrows) was identified by screening using 3D fiesta imaging $(\mathrm{A})$ and it was confirmed by Gd enhanced T1-weighted imaging (B). 3D: three-dimensional.
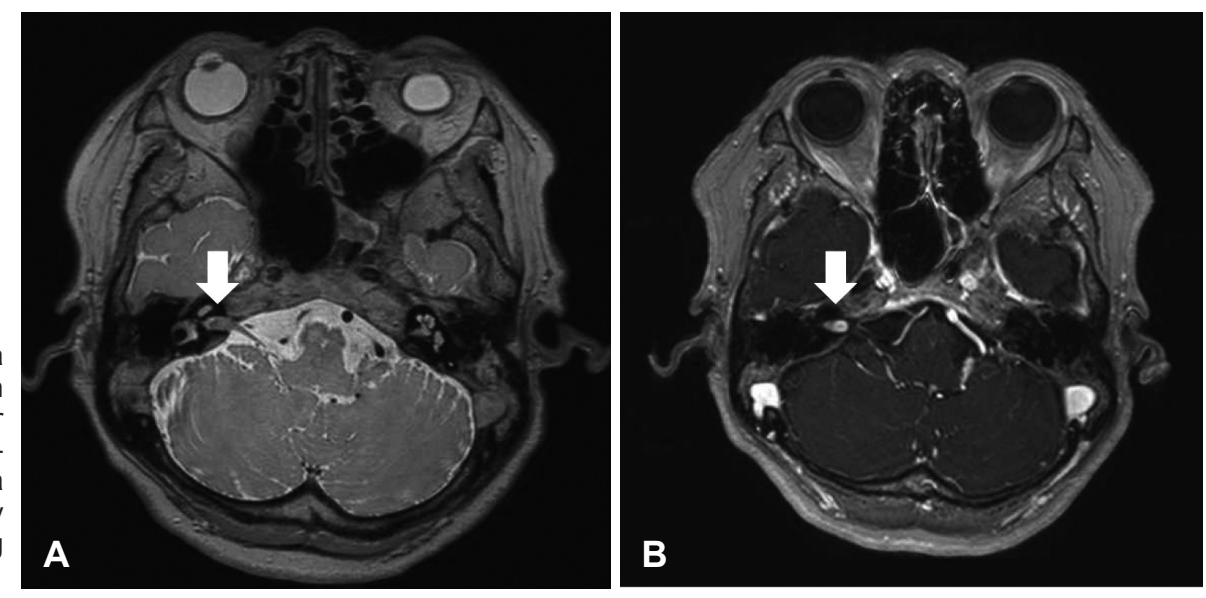

Fig. 4. Temporal bone images of a 49 year-old man with right tinnitus. $A$ tiny fusiform acoustic neuroma (arrows) was identified by screening with 3D fiesta imaging (A) and was confirmed by Flair vista MR imaging (B). 3D: three-dimensional.
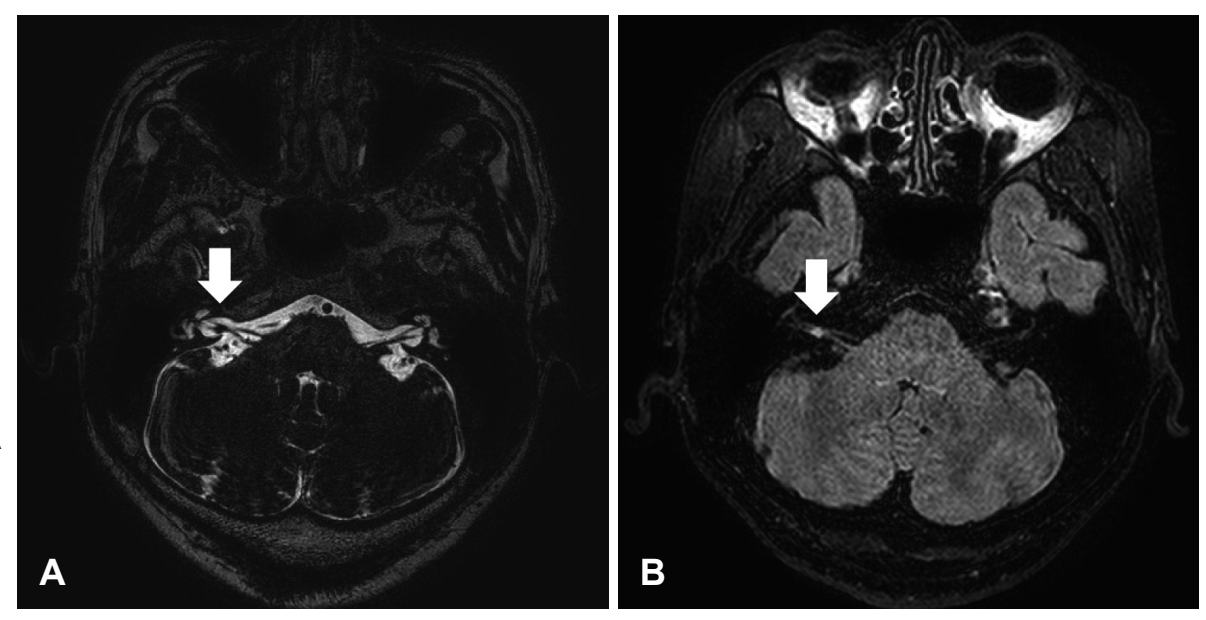

Fig. 5. Temporal bone images of a 38 year-old woman with left sensorineural hearing loss. Enlarged vestibular aqueduct syndrome (arrows) was identified by $3 \mathrm{D}$ fiesta screening $(A)$ and confirmed by temporal bone computed tomography (B). 3D: three-dimensional.
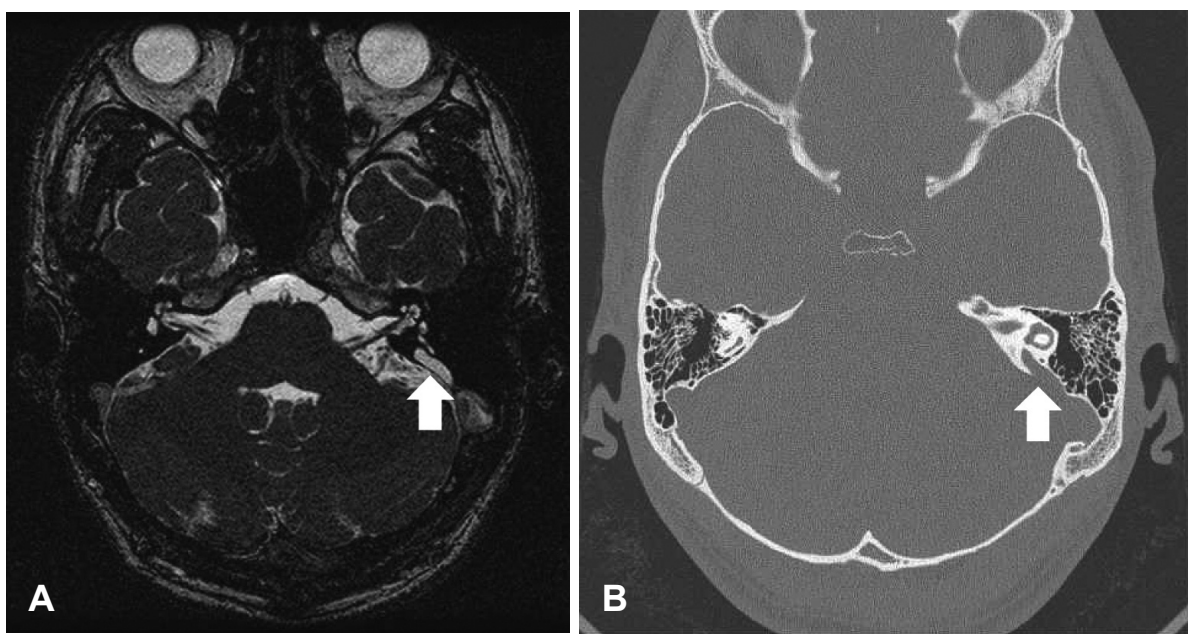

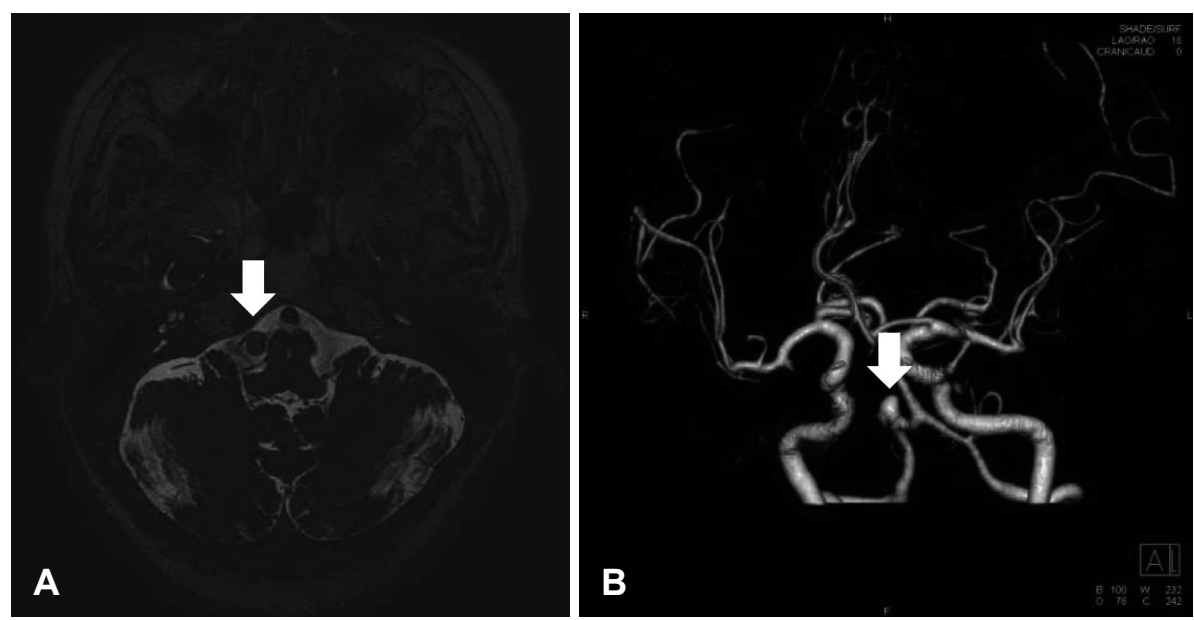

Fig. 6. Temporal bone image of a 69 year-old woman with bilateral continuous tinnitus. Posterior inferior cerebellar artery aneurysm (arrows) was identified by $3 \mathrm{D}$ fiesta screening (A) and confirmed by computed tomography angiography (B). 3D: threedimensional.
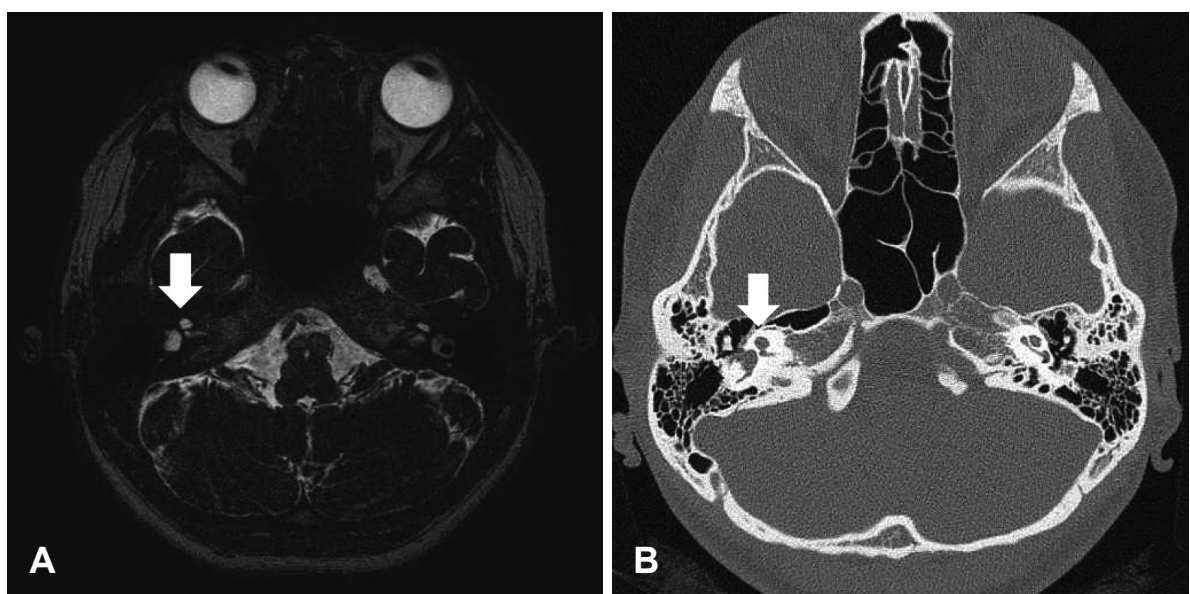

Fig. 7. Temporal bone images of a 16 year-old woman with right sensorineural hearing loss. Mondini dysplasia (arrows) was identified by 3D fiesta screening $(A)$ and confirmed by temporal bone computed tomography (B). 3D: three-dimensional.

was $13 \times 10 \mathrm{~mm}, 52 \times 23 \mathrm{~mm}$, and $11 \times 9 \mathrm{~mm}$ respectively. Table 2 shows the clinical characteristics of patients who were finally diagnosed with acoustic neuroma. The mean tumor size of the unilateral hearing loss group tended to be smaller than that of other groups. However, we could not find any significant correlation between the groups $(p=0.582)$.

\section{Discussion}

In patients with unilateral ear symptoms, it is important to approach these patients with a high index of suspicion for cochlear or retrocochlear lesions such as an inner ear anomaly, tumor, vascular or inflammatory lesion.

With conventional audiologic testing, retrocochlear lesions can be suspected by a relatively low speech discrimination score or low score in the short increment sensitivity index test. Retrochoelar lesions were diagnosed more easily after computed tomography $(\mathrm{CT})$ and the ABR test were introduced. ${ }^{6,7)}$ But the temporal bone CT scan is inadequate for screening tool due to the high false or negative positive rate for the diagnosis of retrocochlear lesions especially in the case of acous- tic neuroma. ABR test has been used as a favored screening tool for retrocochlear lesions; this test compares the amplitude and latency of waves produced by the auditory pathway. However, Barrs, et al. ${ }^{8)}$ reported that even though ABR demonstrated $97 \%$ of the tumors larger than $1.5 \mathrm{~cm}, 48 \%$ of neuromas are $1.5 \mathrm{~cm}$ or smaller. Ruckenstein, et al. ${ }^{9)}$ reported that the ABR screening test for retrocochlear pathology had a sensitivity of $63 \%$ and a specificity of $64 \%$. These findings imply that ABR probably has a limited role in acoustic neuroma screening.

After the application of MRI for the diagnosis of retrocochlear lesions began in the 1980s, the specificity and sensitivity of MRI was found to be up to $100 \%$ and it became to be considered the most accurate diagnostic tool. ${ }^{10,11)}$ But MRI is a very expensive test and it takes 40-60 minutes to get images with contrast. Thus, MRI has limitations as a screening test due to the low cost and time effectiveness.

With the development of the image acquisition technique with MRI, new protocols for 3D-FIESTA sequences were introduced which provided much higher spatial resolution and clearer depiction of small structures such as cranial nerves 
especially within the cisternal spaces. The 3D-FIESTA uses an ultrafast pulse sequence that produces $0.3 \mathrm{~mm}$ thin section high resolution images with outstanding image contrast between the cerebrospinal fluid, vessels and cranial nerves. It usually takes 5 to 7 minutes to get a proper image and does not need contrast media. The cost of $3 \mathrm{D}$-FIESTA is just $1 / 5-1 / 6$ that of conventional MRI. So it could be a very effective test protocol for the screening of retrocochlear lesions in patients
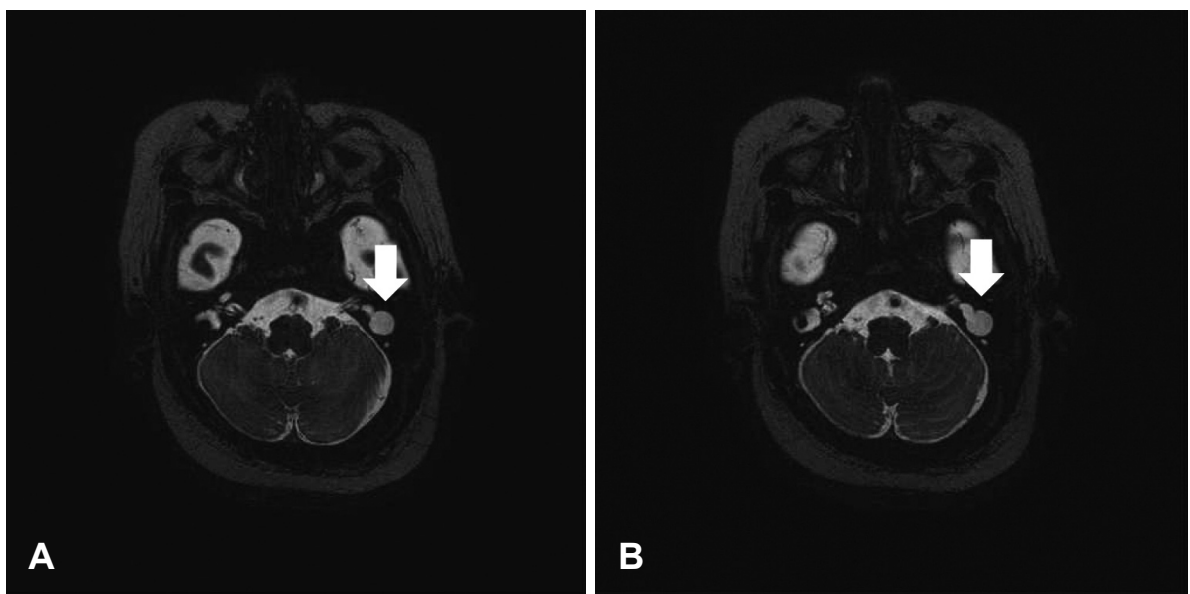

Fig. 8. Temporal bone images of a 1 month-old baby. Left internal auditory canal stenosis with labyrinthine malformation (arrows) was identified by $3 \mathrm{D}$ fiesta screening $(\mathrm{A}-\mathrm{C})$ and confirmed by temporal bone computed tomography (D). 3D: three-dimensional.
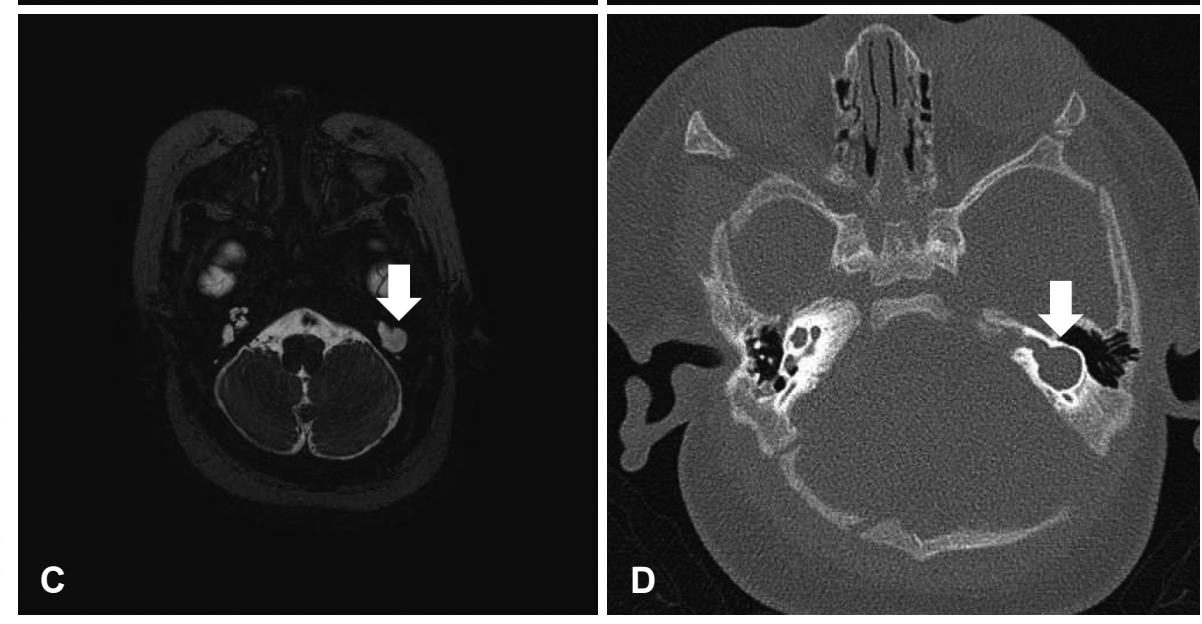

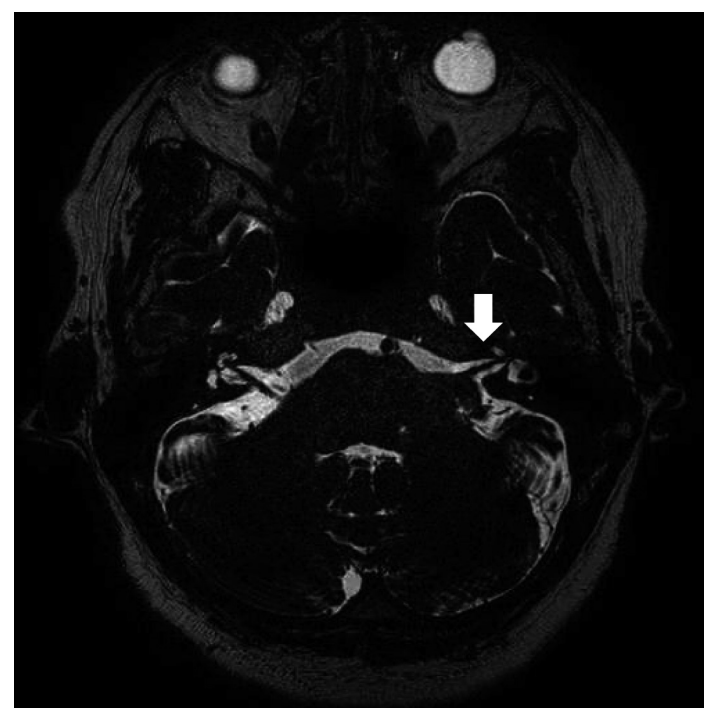

Fig. 9. Temporal bone image of a 51 year-old woman with left sensorineural hearing loss. Hypoplastic vestibulocochlear nerve (arrow) was identified by 3D fiesta screening. 3D: three-dimensional.

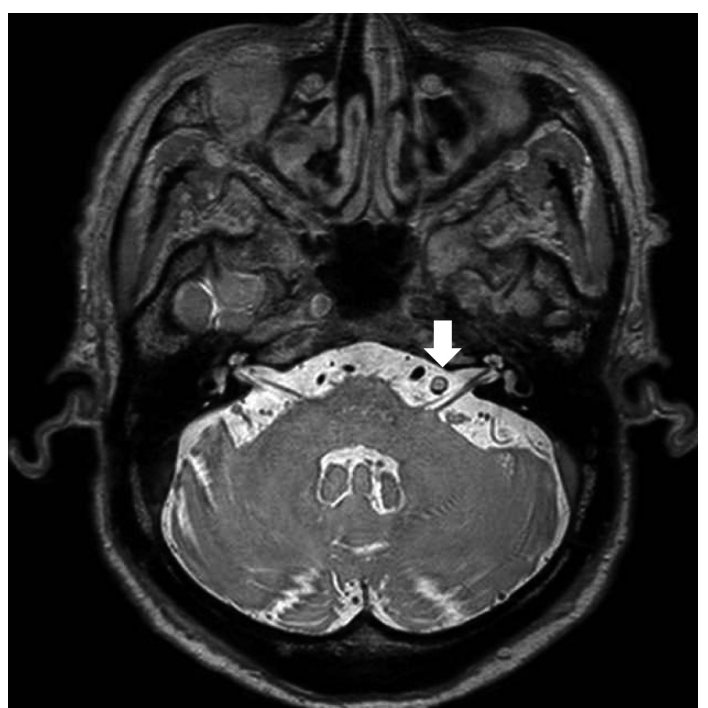

Fig. 10. Temporal bone image of a 76 year-old man with left sudden sensorineural hearing loss. Vertebral artery calcification (arrow) was identified by 3D fiesta screening. 3D: three-dimensional. 
with unilateral ear symptoms.

In this study, variable lesions such as small tumors less than $5 \mathrm{~mm}$, inner ear anomalies and vascular malformations could be identified using 3D-FIESTA imaging. And there is one report which showed that with 3D-FIESTA, there was decreased vestibular signal intensity on the affected side in patients with vestibular schwannoma, but that this was not present in those

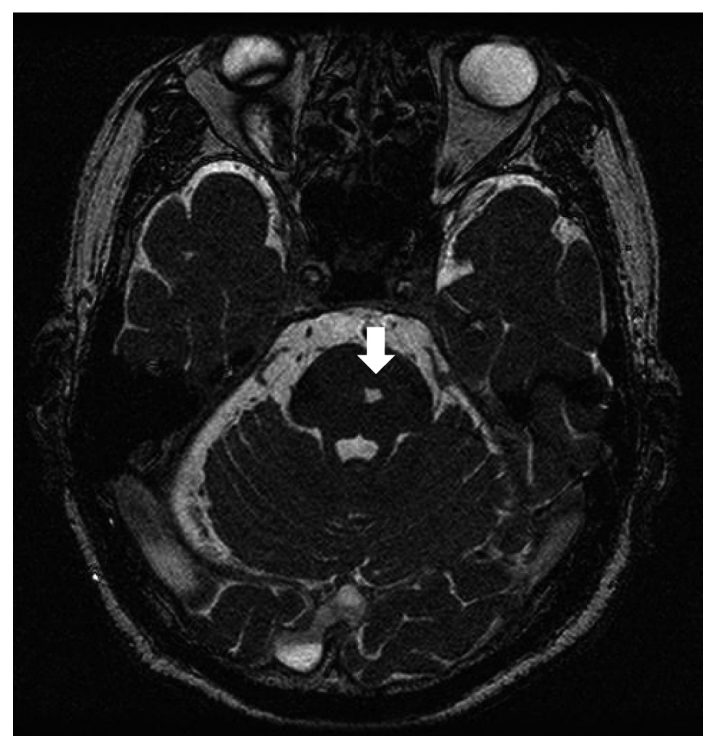

Fig. 11. Temporal bone image of a 73 year-old man with left tinnitus. Brain stem lesion, upper pons infarction (arrow) was identified by 3D fiesta screening. 3D: three-dimensional. with cerebellopontine angle meningiomas or in normal subjects. $^{12)}$

Recently, MRI with high-dose contrast media has been introduced for detecting cochlear endolymphatic hydrops. ${ }^{13)} 3 \mathrm{D}$ FIESTA images have a limitation in detecting inner ear lesions; however, 3D-FIESTA images can detect inner ear anomalies so it may be sufficient to use as a screening tool for retrocochlear lesions.

Acoustic neuroma, one of the most common retrocochlear

Table 2. Summary of acoustic neuroma patients

\begin{tabular}{ccccc}
\hline $\begin{array}{c}\text { Case } \\
\text { No. }\end{array}$ & Sex & $\begin{array}{c}\text { Age } \\
\text { (years) }\end{array}$ & $\begin{array}{c}\text { Chief } \\
\text { complaint* }\end{array}$ & $\begin{array}{c}\text { Tumor } \\
\text { size }(\mathrm{mm})\end{array}$ \\
\hline 1 & $\mathrm{~F}$ & 63 & $\mathrm{~S}$ & $8 \times 4$ \\
2 & $\mathrm{M}$ & 37 & $\mathrm{SS}$ & $10 \times 5$ \\
3 & $\mathrm{M}$ & 35 & $\mathrm{~S}$ & $12 \times 5$ \\
4 & $\mathrm{~F}$ & 67 & $\mathrm{~S}$ & $21 \times 17$ \\
5 & $\mathrm{~F}$ & 56 & $\mathrm{SS}$ & $12 \times 12$ \\
6 & $\mathrm{M}$ & 50 & $\mathrm{~T}$ & $10 \times 9$ \\
7 & $\mathrm{~F}$ & 69 & $\mathrm{~T}$ & $12 \times 6$ \\
8 & $\mathrm{M}$ & 64 & $\mathrm{~S}$ & $62 \times 64$ \\
9 & $\mathrm{~F}$ & 22 & $\mathrm{~T}$ & $30 \times 28$ \\
10 & $\mathrm{M}$ & 36 & $\mathrm{~T}$ & $10 \times 6$ \\
11 & $\mathrm{~F}$ & 56 & $\mathrm{~T}$ & $11 \times 8$ \\
12 & $\mathrm{M}$ & 49 & $\mathrm{~T}$ & $4 \times 3$ \\
\hline
\end{tabular}

*chief complaint. S: sensorineural hearing loss, SS: sudden sensorineural hearing loss, T: tinnitus
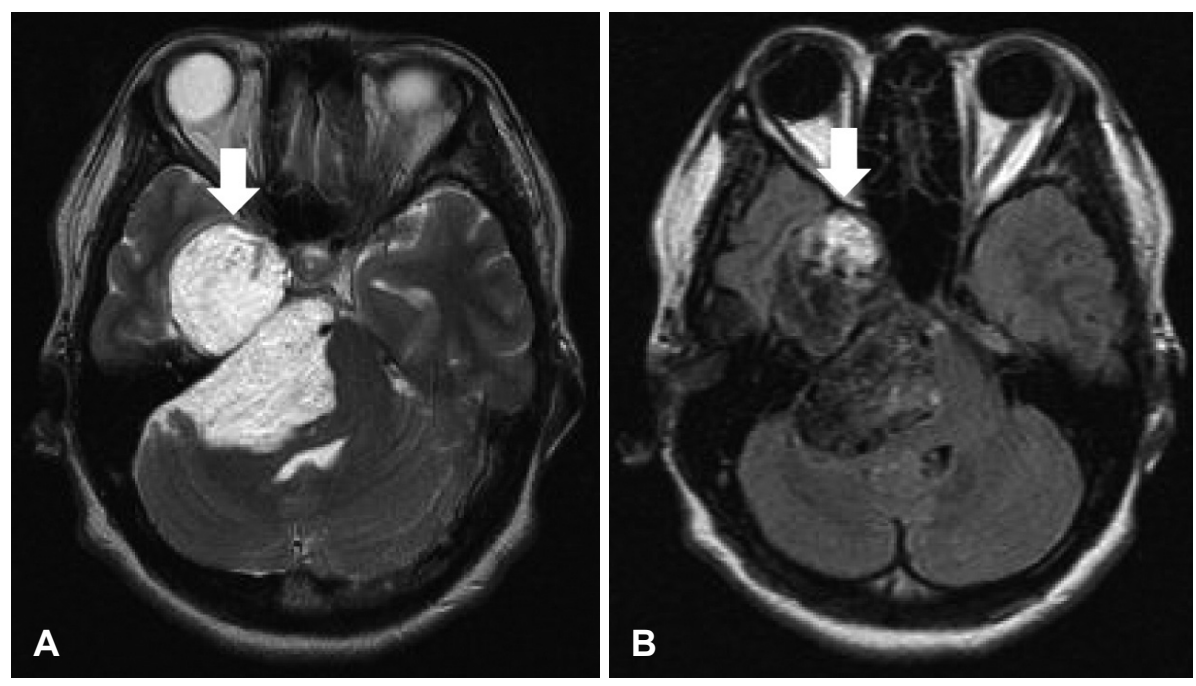

Fig. 12. Temporal bone images of a 51 year-old man with right sensorineural hearing loss. Huge epidermal cyst (arrows) was identified by $3 \mathrm{D}$ fiesta screening $(A)$ and confirmed by Gd enhanced T1-weighted imaging (B). 3D: three-dimensional.

Table 1. Disease distribution and ratio according to the cause

\begin{tabular}{|c|c|c|c|c|c|}
\hline Chief complaint & Acoustic neuroma & EVAS & PICA aneurysm & Inner ear anomaly & 8th n. hypoplasia \\
\hline Tinnitus (129) & $6(4.6 \%)$ & & $2(1.5 \%)$ & & \\
\hline SSNHL (83) & $2(2.4 \%)$ & $1(1.2 \%)$ & & & $1(1.2 \%)$ \\
\hline Unilat. HL (41) & $4(9.7 \%)$ & $3(7.3 \%)$ & & $2(4.8 \%)$ & $1(2.4 \%)$ \\
\hline
\end{tabular}

SSNHL: sudden sensorineural hearing loss, HL: hearing loss, EVAS: enlarged vestibular aqueduct syndrome, PICA: posterior inferior cerebellar artery 
lesions, is a benign tumor originating from the vestibular nerve sheath in the internal auditory canal. In the early stage of acoustic neuromas, symptoms can be nonspecific but as the tumor grows, progressive unilateral hearing loss and tinnitus are typical symptoms. It is well known that about 10 to $20 \%$ of acoustic neuroma patients can be present with sudden sensorineural hearing loss, and on the other hand only 1 to $3 \%$ of sudden seonsorineural hearing loss patients have acoustic neuro$\mathrm{ma}^{14-16)}$

In this study, 9.7\% of unilateral hearing loss patients, $4.6 \%$ of unilateral tinnitus and $2.4 \%$ of sudden sensorineural hearing loss patients were diagnosed with acoustic neuroma. The mean size of acoustic neuroma varied from a tiny tumor smaller than $5 \mathrm{~mm}$ to a large tumor up to $64 \mathrm{~mm}$. The mean size of the tumor in the sudden hearing loss patients group was larger than the other two groups, but there was no statistically significant difference between the groups. In addition to acoustic neuroma, many retrocochlear lesions, intracranial masses and acute infarctions can be diagnosed with screening using 3DFIESTA imaging.

With these findings, we suggest that 3D-FIESTA imaging could be a reliable screening test for patients who complain of unilateral otologic symptoms. But considering that this study was a simple retrospective observational study, further comparative study investigating the sensitivity and specificity of 3D-FIESTA imaging will be required.

\section{Conclusion}

3D-FIESTA imaging is a cost-effective and sensitive method for the diagnosis of cochlear or retrocochlear lesions. 3DFIESTA imaging is a useful screening tool for patients with unilateral ear symptoms.

\section{REFERENCES}

1) Kim H, Lee YS, Chung JW, Yoon TH, Lee KS. Efficacy of audiologic tests in the differential diagnosis of cochlear and retrocochlear hearing loss. Korean J Otolaryngol-Head Neck Surg 1999;42:955-60.

2) Cueva RA. Auditory brainstem response versus magnetic resonance imaging for the evaluation of asymmetric sensorineural hearing loss. Laryngoscope 2004;114:1686-92.

3) Wilson DF, Hodgson RS, Gustafson MF, Hogue S, Mills L. The sensitivity of auditory brainstem response testing in small acoustic neuromas. Laryngoscope 1992;102:961-4.

4) Erdogan N, Altay C, Akay E, Karakas L, Uluc E, Mete B, et al. MRI assessment of internal acoustic canal variations using 3D-FIESTA sequences. Eur Arch Otorhinolaryngol 2013;270:469-75.

5) Rigby PJ. Comparison of FIESTA and gadolinium-enhanced T1weighted sequences in magnetic resonance of acoustic schwannoma. Radiographer 2006;53:11-21.

6) Selters WA, Brackmann DE. Acoustic tumor detection with brain stem electric response audiometry. Arch Otolaryngol 1977;103:181-7.

7) House JW, Waluch V, Jackler RK. Magnetic resonance imaging in acoustic neuroma diagnosis. Ann Otol Rhinol Laryngol 1986;95(1 Pt 1):16-20.

8) Barrs DM, Brackmann DE, Olson JE, House WF. Changing concepts of acoustic neuroma diagnosis. Arch Otolaryngol 1985;111:1721.

9) Ruckenstein MJ, Cueva RA, Morrison DH, Press G. A prospective study of $A B R$ and MRI in the screening for vestibular schwannomas. Am J Otol 1996;17:317-20.

10) Jackler RK, Shapiro MS, Dillon WP, Pitts L, Lanser MJ. Gadolinium-DTPA enhanced magnetic resonance imaging in acoustic neuroma diagnosis and management. Otolaryngol Head Neck Surg 1990; 102:670-7.

11) Glasscock ME 3rd, Hays JW, Minor LB, Haynes DS, Carrasco VN. Preservation of hearing in surgery for acoustic neuromas. J Neurosurg 1993;78:864-70.

12) Ishikawa K, Haneda J, Okamoto K. Decreased vestibular signal intensity on 3D-FIESTA in vestibular schwannomas differentiating from meningiomas. Neuroradiology 2013;55:261-70.

13) Seo YJ, Kim J, Choi JY, Lee WS. Visualization of endolymphatic hydrops and correlation with audio-vestibular functional testing in patients with definite Meniere's disease. Auris Nasus Larynx 2013; 40:167-72.

14) Morrison GA, Sterkers JM. Unusual presentations of acoustic tumours. Clin Otolaryngol Allied Sci 1996;21:80-3.

15) Sataloff RT, Davies B, Myers DL. Acoustic neuromas presenting as sudden deafness. Am J Otol 1985;6:349-52.

16) Lee YW, Lee KD, Yu TH, Kim HK. Neurotologic analysis of cerebellopontine angle tumor. Korean J Otolaryngol-Head Neck Surg 1998;41:454-60. 